\title{
分子内氧化偶联反应在合成复杂吲哚生物碱骨架中的应用
}

\author{
谢卫青*, $a$ 左智伟 ${ }^{a}$ 资伟伟 ${ }^{a}$ 马大为 $*, a$ \\ $\left({ }^{a}\right.$ 中国科学院上海有机化学研究所 生命有机化学国家重点实验室 上海 200032)
}

\begin{abstract}
摘要 通过含有啊哚底物的分子内氧化偶联反应, 成功地构建了 Communesin 家族生物碱的螺呵哚啉季碳中心, 从而 完成了(一)-Communesins A, B 和 $\mathrm{F}$ 的对映选择性合成. 接下来我们发展了分子内氧化偶联/缩合串联反应策略，得到了 天然产物(一)-Vincorine 的核心四环骨架, 然后再经过五步转化完成了 Vincorine 的全合成. 从药物化学角度来看, 分子 内氧化偶联/缩合串联提供了一个快速方便地合成含有多环吲哚啉骨架的方法. 采用相同的串联反应策略, 我们分别从 色胺衍生的 $\beta$-酮酸酰胺和丙二酸二酰胺出发, 一步构建了多环螺呵哚啉和多环品哚啉并吡咯环骨架分子.

关键词 分子内氧化偶联; 吲哚生物碱; 全合成; 多环螺吲哚啉; 吲哚并吡咯环
\end{abstract}

\section{Intramolecular Oxidative Coupling: Applications in Synthesis of Complex Indole Akaloid Scaffolds}

\author{
Xie, Weiqing ${ }^{*, a} \quad$ Zuo, Zhiwei $^{a} \quad$ Zi, Weiwei ${ }^{a} \quad$ Ma, Dawei*,a \\ ( ${ }^{a}$ State Key Laboratory of Bioorganic and Natural Products Chemistry, Shanghai Institute of Organic Chemistry, \\ Chinese Academy of Science, Shanghai 200032)
}

\begin{abstract}
Intramolecular oxidative coupling of tryptamine incorporated amide was used to create the quaternary spiroindoline carbon center of communesins, which enabled a short asymmetric synthesis of (-)-communesins A and B and F. The fused tetra-ring framework of Vincorine was established by an intramolecular oxidative coupling/condensative cyclization process, which was further advanced to ( - -)vincorine in 5 steps. From a medicinal standpoint, such a cascade process provides a highly diverse, efficient method for the construction of polycyclic spiroindoline scaffolds. Starting from easily accessible tryptamine incorporated $\beta$-ketoamides and malonamides, polyclic spiroindolines and pyrroloindolines could be directly obtained by adopting the same cascade strategy.
\end{abstract}

Keywords intramolecular oxidative coupling; indole alkaloids; total synthesis; polycyclic spiroindoline; pyrroloindoline

烯醇负离子氧化偶联反应是一种高效的形成碳碳 键反应 ${ }^{[1,2]}$. 它不需要预先对反应底物进行官能团化, 仅 仅通过强碱将羰基化合物去质子化形成烯醇负离子，然 后加入氧化剂就可以将两个羰基 $\alpha$ 位碳连接起来,一步 得到 1,4 二羰基化合物. 从合成经济角度来看, 由于不 需要预先对参与反应的碳原子进行官能团化, 从而可以 有效地提高一个全合成的效率. 虽然早在 1935 年 Ivanoff 等 ${ }^{[1 a]}$ 就发现了这一反应, 但在之后的 70 年中这 一类反应始终没有在合成复杂天然产物得到应用. 究其 原因在于不同分子间的烯醇负离子氧化偶联时往往存 在较多的竞争反应(如同分子的氧化偶联反应, Claisen 缩合反应及卤代反应), 从而限制了氧化偶联反应在有
机合成中的应用.

直到 2004 年, Baran 等 ${ }^{[3 a, 3 c]}$ 在对吲哚萜类生物碱全 合成研究中发现, 吲哚作为烯胺也可以参与分子间的氧 化偶联反应. 通过吲哚和羰基化合物的烯醇负离子氧化 偶联实现了羰基 $\alpha$ 位的直接吲哚化. 正是基于这一反应, Baran 小组 ${ }^{[3]}$ 完成了一系列复杂吲哚生物碱的全合成, 如 Hapalindole 家族生物碱, Fisherindole 家族生物碱及 Welwitindolinone 的全合成. 为了克服分子间偶联反应 的复杂性，提高反应的选择性，另外一种策略是采用分 子内氧化偶联的方法. 这样不但可以提高反应的收率, 同时能够构建复杂环系骨架. 正是基于这一思路, Baran 等 ${ }^{[4]}$ 通过分子内氧化偶联反应，构建了合成 Stephacidin

\footnotetext{
*E-mail: xiewq@sioc.ac.cn; madw@sioc.ac.cn

Received January 15, 2013; revised January 31, 2013; published online February 1, 2013.

Project supported by the National Basic Research Program of China (973 Program, No. 2010CB833200).

国家重点基础研究发展计划(973 计划, No. 2010CB833200)资助项目.
} 
的关键的桥环中间体. 而 Overman 小组 ${ }^{[5]}$ 在合成 Actinophyllic acid 中, 也是基于分子内氧化偶联反应构 建了合成的关键的桥环中间体, 最后高效地完成了对 Actinophyllic acid 的全合成. 从两个合成来看, 分子内 氧化偶联反应在构建多环骨架起到了重要的作用. 而且 从合成策略上来看, 分子内氧化偶联反应的应用有效地 提高了整个合成的效率.

我们小组 ${ }^{[6]}$ 通过设计含有吲哚的关环前体, 以分子 内的氧化偶联反应为关键合成步骤完成了对 (一)-Communesins A, B 和 F 的对映选择性合成, 并由此 确定了天然产物的绝对构型(图 1). 而通过采用分子内 氧化偶联、关环串联反应策略, 完成了复杂天然生物碱 (一)-Vincorine 的全合成 ${ }^{[7]}$. 由于这些天然产物的合成路 线比较长, 从药物化学角度来说, 也制约了对这些天然 产物进行构效关系的研究. 我们运用分子内氧偶联、关 环串联反应, 从简单的底物出发, 一步构建了多环螺吲 哚啉骨架 ${ }^{[8]}$ 和多环吡咯并吲哚啉骨架 ${ }^{[9]}$. 得到的多环骨 架分子和已知天然产物有一定的相似性, 可以推断这一 类产物可能表现出较好的生物活性. 而且由于底物合成 简单，从而我们可以对这些多环骨架的产物进行系统的 构效关系的研究.
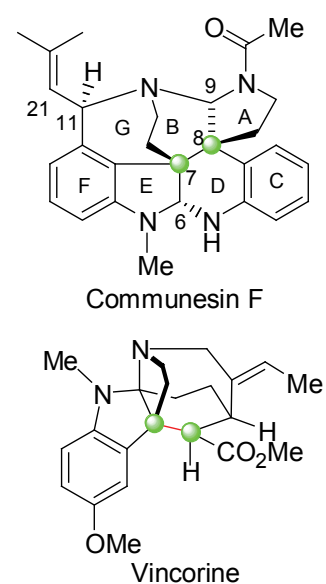

Vincorine
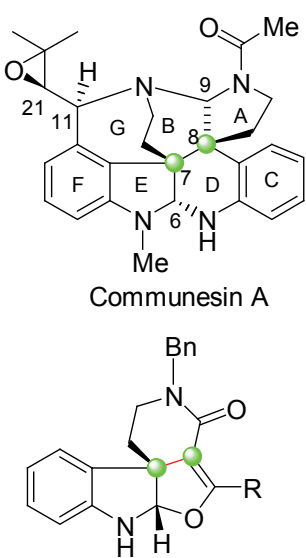

polycyclic sipiroindolines<smiles>[R]C1C(=O)N([R])C(=O)C12Nc1ccccc1NCCO2</smiles>

polycyclic pyroloindolines

图 1 应用分子间氧化偶联反应构建的复杂生物碱骨架

Figure 1 Complex alkaloid scaffolds created by employing intramolecular oxidative coupling as key step

\section{1 分子内氧化偶联反应在 Communesin 家族 生物碱合成中的应用}

1993 年, Numata 等 ${ }^{[10 a]}$ 从生长在海藻(Enteromorpha
intestinalis)上的青霉菌(Penicillium sp.)中分离出两个结 构新颖且具有重要生理活性的生物碱, Communesins A 和 $\mathrm{B}$ (图 2). 在此之后, 与它们结构类似的 Communesin $\mathrm{C} \sim \mathrm{H}$ 被陆续分离并鉴定 ${ }^{[10 \mathrm{~b} ~ 10 \mathrm{~d}]}$. 初步的生物活性测试 表明, 这一系列生物碱均具有较好的抗癌和杀虫活性. 从结构上来看, Communesins $\mathrm{A} \sim \mathrm{H}$ 都具有七环的稠环 结构, 手性中心密集, 含有的两个缩醛胺结构分别位于 分子上下两端, 其中 $\mathrm{C}(7), \mathrm{C}(8)$ 为两个连续的手性季碳. 复杂的稠环结构和密集的手性中心给这类生物碱的合 成带来了很大的挑战，同时也吸引了多个合成小组的兴 趣 $^{[6,11]}$. 在我们的工作完成之前, 有两个小组报道了 $( \pm)$-Communesin F 的全合成 ${ }^{[11]}$.

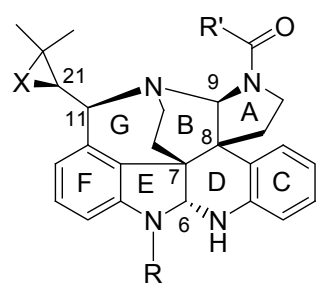

\begin{tabular}{ccccl}
\hline Compd. & Communesin & $\mathrm{X}$ & $\mathrm{R}$ & \multicolumn{1}{c}{$\mathrm{R}^{\prime}$} \\
\hline $\mathbf{1}$ & $\mathrm{A}$ & $\mathrm{O}$ & $\mathrm{CH}_{3}$ & $\mathrm{CH}_{3}$ \\
$\mathbf{2}$ & $\mathrm{B}$ & $\mathrm{O}$ & $\mathrm{CH}_{3}$ & 2,4-Pentadienyl \\
$\mathbf{3}$ & $\mathrm{C}$ & $\mathrm{O}$ & $\mathrm{H}$ & 2,4-Pentadienyl \\
$\mathbf{4}$ & $\mathrm{D}$ & $\mathrm{O}$ & $\mathrm{CHO}$ & 2,4-Pentadienyl \\
$\mathbf{5}$ & $\mathrm{E}$ & $\mathrm{O}$ & $\mathrm{H}$ & 2,4-Pentadienyl \\
$\mathbf{6}$ & $\mathrm{F}$ & $\mathrm{H}, \mathrm{H}$ & $\mathrm{CH}_{3}$ & $\mathrm{CH}_{3}$ \\
$\mathbf{7}$ & $\mathrm{H}$ & $\mathrm{O}$ & $\mathrm{CH}_{3}$ & $\mathrm{CH}_{2} \mathrm{CH}_{3}$ \\
$\mathbf{8}$ & $\mathrm{H}$ & $\mathrm{O}$ & $\mathrm{CH}_{3}$ & $\mathrm{CH}_{2} \mathrm{CH}_{2} \mathrm{CH}_{3}$ \\
\hline
\end{tabular}

图 2 Communesin 家族生物碱的结构

Figure 2 Structures of communesin family

为了使合成更为高效, 我们设想通过 $\mathrm{C}(3)$ 位取代的 吲哚和酰胺 $\alpha$ 位的烯醇负离子的分子内氧化偶联来构建 Communesin 生物碱的 BE 螺环结构(Eq. 1) ${ }^{[6 a]}$. 由于文献 中没有报道过 3 位有取代的吲哚参与氧化偶联反应来构 建季碳中心的先例, 因此我们对模型反应进行了尝试.

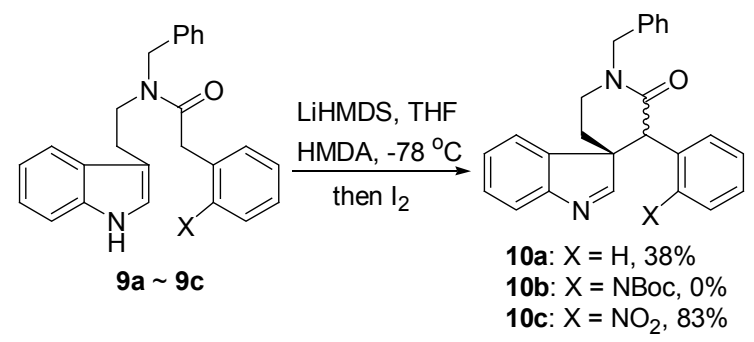

首先我们对酰胺 9a 的分子内氧化偶联反应进行了 研究. 在 LiHMDS 的作用下形成双负离子, $-78{ }^{\circ} \mathrm{C}$ 下 滴加碘, 即可以 $38 \%$ 的收率分离到吲哚啉 $10 a$. 但是遗 
憾的是带有 Boc 保护氨基的酰胺 9b 在此条件下未能发 生反应. 令我们高兴的是, 含硝基的酰胺 $9 \mathrm{c}$ 在该条件下 能以 $83 \%$ 的收率, $2: 1$ 的 $d r$ 值得到吲哚啉 10c. 从这些 数据中可以得出初步的结论: 羰基参与烯醇负离子的氧 化偶联反应活性可能与其活性亚甲基的 $\mathrm{p} K_{\mathrm{a}}$ 的大小有 关.

在模型反应成功之后, 我们展开了对 Communesin $F$ 的合成工作 $\left(\right.$ Scheme 1) ${ }^{[6 a]}$. 为了完成对 Communesin F 对映选择性合成, 我们计划在氧化偶联的底物酰胺上引 入手性辅基来控制生成的两个手性中心的构型. 以 4-溴 色醇为原料, 通过 3 步合成了带有手性辅基的酰胺 11. 在与模型反应相同的条件下氧化偶联得到吲哚啉, 接着 用铁粉还原硝基环化, 然后选择性甲基化得到一对非对 映异构体可以通过柱层析分离. 两个异构体比例约为 3: 1, 主要产物 12 的收率为 $50 \%$, 它的绝对构型通过单 晶衍射确定.

化合物 12 在 $\mathrm{KHMDS} / \mathrm{Boc}_{2} \mathrm{O}$ 的条件对缩醛胺的氮 进行保护. 接着以叔丁醇钾为碱, 烯丙基碘为亲电试剂 引入第二个季碳中心. 结果以 $96 \%$ 的收率, 单一的立体 选择性得到化合物 13. 之后我们通过 Heck 反应引入芳 基侧链, 得到五环化合物 14. 接着在甲磺酰氯促进下形
成 $\mathrm{G}$ 环并通过 Staudinger/Aza-Wittig 反应顺利构建了最 后的 $\mathrm{A}$ 环. 去保护和乙酰化之后, 我们顺利的得到了 (一)-Communesin F. 通过和天然产物的旋光比较, 我们 确定了天然产物绝对构型为 $(6 R, 7 R, 8 R, 9 S, 11 R)$.

在合成了大量的(一)-Communesin $\mathrm{F}$ 后, 我们试图 通过直接对 Communesin $\mathrm{F}$ 环氧化来构建结构最为复杂 的 Communesins A 和 B. 由于分子中存在多个叔胺结构, 我们尝试了多种氧化剂, 但均未能取得成功. 于是我们 决定放弃直接转化的方法, 转而在合成的早期在侧链引 入二醇结构, 发展一条新路线来合成含有环氧官能团的 Communesins $\mathrm{A}$ 和 $\mathrm{B}^{[6 \mathrm{~b}]}$.

含有缩酮保护的邻二醇单元的仲胺 16 在 $\mathrm{BOPCl}$ 的 促进下与邻硝基苯乙酸缩合，以 $91 \%$ 的收率得到了酰胺 17 (Scheme 2). 参照之前的条件, 以 LiHMDS 为碱, THF 为溶剂的条件下, 化合物 17 形成双负离子. 在 $-78{ }^{\circ} \mathrm{C}$ 下加入碘溶液然后升至室温反应时, 生成了大量的碘代 物 20 (以一对非对映异构体存在). 我们试图将碘代产物 20 在强碱如 LiHMDS 的作用下通过 $\mathrm{S}_{\mathrm{N}} 2$ 机理形成关环 产物 19. 但是一旦升温反应体系就变得异常复杂, 没能 检测到任何桥环产物 19 的生成. 经过研究我们发现,<smiles>CC(C)(C)OC[C@H](c1ccccc1)N(CCc1ccccc1Br)C(=O)Cc1ccccc1[N+](=O)[O-]</smiles>

(1) LiHMDS, $\mathrm{I}_{2}, \mathrm{THF},-78^{\circ} \mathrm{C} \mathrm{Br}$ (2) $\mathrm{Fe} / \mathrm{NH}_{4} \mathrm{Cl}, t-\mathrm{BuOH} / \mathrm{H}_{2} \mathrm{O}$ (3) KHMDS, Mel dr 3:1, major isomer: $50 \%$ for three steps

11<smiles>CN(c1cccc(/C=C/C(C)(C)O)c1)[C@@H]1[C@H]2CCNC(=O)[C@@]2(CCO)c2ccccc2[C@H]1C(C)(C)C</smiles><smiles>C=CC#CC=C</smiles>

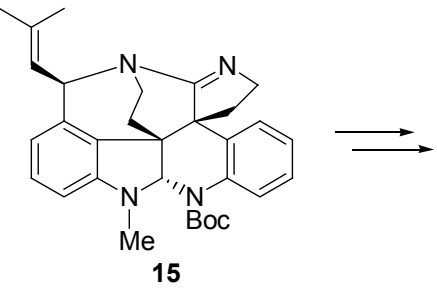

(1) $\mathrm{KHMDS},(\mathrm{Boc})_{2} \mathrm{O}$

(2) KOBu-t, allyl iodide $87 \%$ for two steps

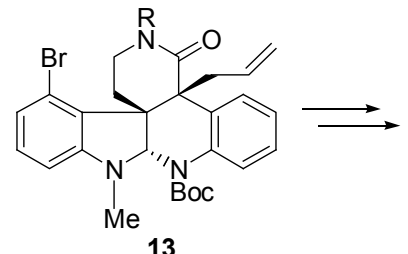
13

Scheme 1
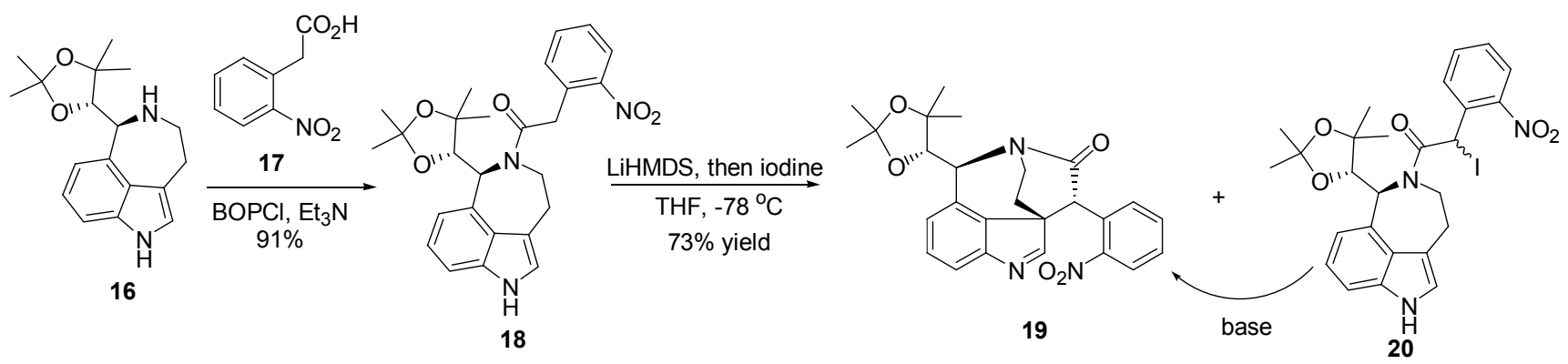

Scheme 2 
将低温下生成的双负离子升至室温后再滴加碘溶液，15 $\min$ 后停止反应, 能以 $66 \%$ 的收率分离到螺环吲哚啉 19(基于回收原料的收率为 73\%).

含有螺吲哚林环结构的化合物 19 通过还原环化, 接着甲基化得到缩醛胺 21. 由于分子的位阻增大的原 因, 采用之前烯丙基化条件来构建 $\mathrm{C}(8)$ 位季碳中心的方 法完全不能得到产物. 我们转而采用活性更高的烷基化 试剂碘乙腈, 结果能以 $67 \%$ 收率得到烷基化产物 22. 最 后我们需要关上 $\mathrm{A}$ 环, 并在氮上引入酰基. 经过多次失 败的尝试, 我们发现 $\mathrm{A}$ 环可以通过两步串联反应形成: $\mathrm{LiAlH}_{4}$ 同时还原扭曲酰胺和腈基得到半缩醛, 其后在 $\mathrm{NH}_{4} \mathrm{OAc} / \mathrm{NaBH}(\mathrm{OAc})_{3}$ 的条件下形成缩醛胺. 化合物 23 通过酰化引入侧链, 脱除缩酮保护后通过 $\mathrm{S}_{\mathrm{N}} 2$ 反应即可
构建环氧，顺利的合成了(一)-Communesins A 和 B (Scheme 3).

\section{2 通过分子内氧化偶联反应合成 Vincorine}

吲哚类生物碱 Akuammiline 从生源上讲是生物碱 Geissoschizine 的 C(7)和 C(16) 关环衍生出来的一系列生 物碱(图 3) ${ }^{[12]}$. Vincorine ${ }^{[13]}$ 是 Akuammiline 家族中的代表 化合物. 和其它 Akuammiline 生物碱诸如 Aspidophylline

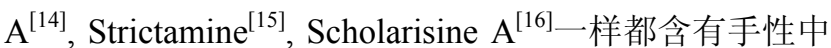
心富集的一个五环形成的稠环结构, 一个螺吲哚啉季碳 中心. 由于它们结构的复杂性以及优异的生物活性, 吸 引了众多合成小组的兴趣 ${ }^{[17]}$.

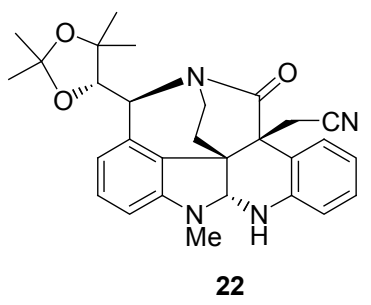

(1) $\mathrm{LAH}, \mathrm{THF}$

(2) $\mathrm{NH}_{4} \mathrm{OAc}, \mathrm{NaBH}(\mathrm{OAc})_{3}$

$92 \%$ for 2 steps
HF, $-78{ }^{\circ} \mathrm{C} \sim$ r.t.

$67 \%$

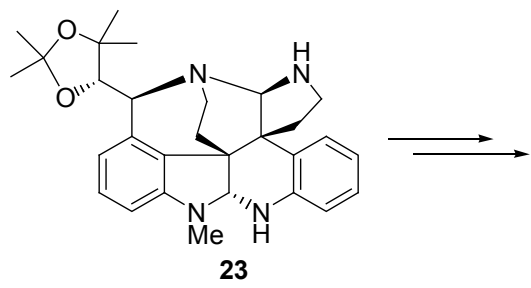<smiles>[R]C(=O)N1CC[C@]23c4ccccc4N[C@H](N(C)c4cccc(C5OC5(C)C)c4)[C@@]12CCN3C</smiles>

(-)-Communesin $\mathrm{A}, \mathrm{R}=\mathrm{CH}_{3}$ (-)-Communesin $\mathrm{B}, \mathrm{R}=$ 2,4-pentadienyl

Scheme 3

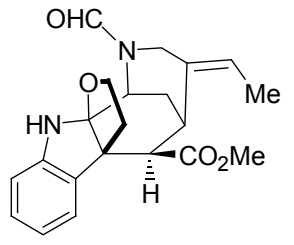

Aspidophylline A

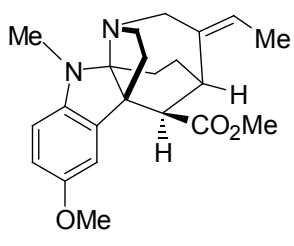

Vincorine

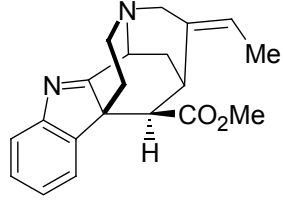

Strictamine

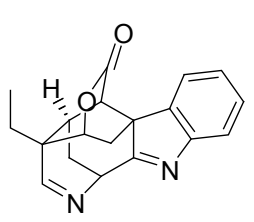

Scholarisine A

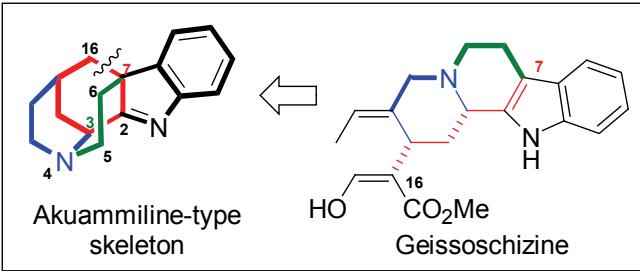

图 3 Akuammiline 家族代表性生物碱及其特征骨架结构

Figure 3 Representative akuammiline alkaloids and Akuammiline-type skeleton 
基于分子内氧化偶联反应在合成吲梠生物碱的高 效性, 我们开始了对 Vincorine 的合成研究(Scheme 4) ${ }^{[7]}$. 为了完成对 Vincorine 的不对称合成, 我们采用小分子 催化的不对称 Michael 加成反应来构建 Vincorine C(4) 位的手性中心. 从 5-甲氧基吲哚出发, 经过几步转化得 到 Michael 加成前体 25. 通过有机小分子催化的醛 $\mathbf{2 6}$ 和不饱和酯 $\mathbf{2 5}$ 的 Michael 加成反应得到化合物 $\mathbf{2 7}$, 两个 异构体比例为 $5: 1$. 然后经过几步官能团转化得到了 氧化偶联关环前体 28 . 由于醛 $27 \alpha$ 位手性被消除, 最后 得到的产物 28 的 $e r$ 值只有 $82: 18$. 化合物 $\mathbf{2 8}$ 在 -40 ${ }^{\circ} \mathrm{C}$ 下用 2 equiv.的 LHMDS 擢氢, 然后加入碘作为氧化 剂, 缓慢升至室温, 即可以 $67 \%$ 的收率得到氧化偶联的 产物 29. 该反应一步构建了 Vincorine 骨架中的两个环, 而且得到季碳的构型也与天然产物中一致. 之后通过 Krapcho 脱羧反应, 脱除硅保护基后将烯丙醇氯代, 得 到化合物 30. 最后脱除仲胺的 Boc 保护, 通过分子内烷 基化反应形成了 Vincorine 的最后一个七元环. 甲基化 后即顺利完成了(一)-Vincorine 的全合成.

\section{3 分子内氧化偶联启动的串联反应在构建多环 吲哚啉骨架中的应用}

虽然我们运用分子内氧化偶联反应为关键步骤完 成了复杂生物碱 Communesin 及 Vincorine 的全合成 ${ }^{[6,7]}$, 但从药物化学的角度来讲, 合成这些生物碱的合成步骤
太长，从而限制了对这两类生物碱进行构效关系研究. 由于多环吲哚生物碱一般都有较好的生物活性, 因此发 展出一条高效的构建多环吲哚生物碱骨架方法有助于 活性化合物的笁选. 在 Vincorine 的合成中，我们通过分 子内的氮原子捕获分子内氧化偶联生成的亚胺中间体, 从而一步构建了四环体系. 我们设想如 Scheme 5 所示, 如果在酰胺 31 中还存在另外一个亲核基团, 则在分子 内氧化偶联反应之后得到亚胺 32, 亲核试剂进攻亚胺 就得到了三环化合物 33. 这样通过在酰胺中引入亲核 基团我们就可以一步从简单酰胺化合物 31 合成多环吲 哚啉骨架 33. 而且 33 可以进一步进行结构修饰从而得 到一些结构新颖得吲哚生物碱化合物. 由于直链酰胺 31 可以很方便地从色胺经过两步反应得到, 因此通过 这样的串联反应可以快速得到一类含有多环吲哚啉生 物碱分子库, 从而为这类化合物的构效关系的研究打下 坚实的基础.

我们首先对 $\beta$-酮酸酰胺 34 的氧化偶联进行研究 ${ }^{[8]}$. 我们的设想是，在分子内氧化偶联反应之后，由于 $\beta$-酮 酸酰胺 34 羰基容易烯醇化而且烯醇氧原子有一定的亲 核性, 这样烯醇氧原子进攻亚胺关环就得到了多环的螺 吲哚环产物.

经过条件的优化，我们发现用只有 $\mathrm{I}_{2}$ 作氧化剂才可 以较好收率得到产物，而金属氧化剂(二价铜盐和三价 铁盐)则不能够促进反应的发生. 得到最优条件之后,<smiles>CC#CCNCCc1[nH]c2ccc(OC)cc2c1CCCN</smiles>

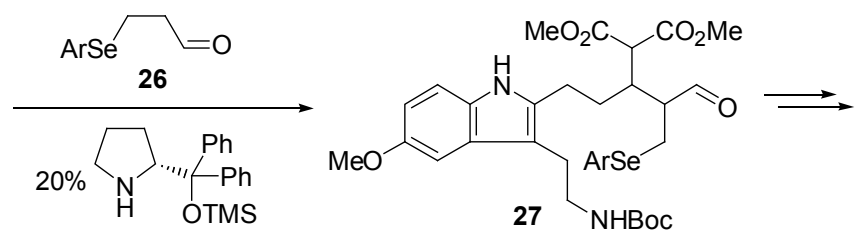

$20 \% \mathrm{HOAc}, \mathrm{CH}_{3} \mathrm{CN}, 0^{\circ} \mathrm{C}$

$75 \%$, dr $5: 1$

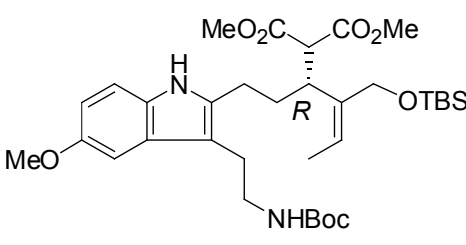

28

er 82:18

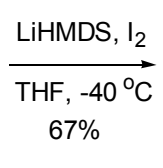

$7 \%$

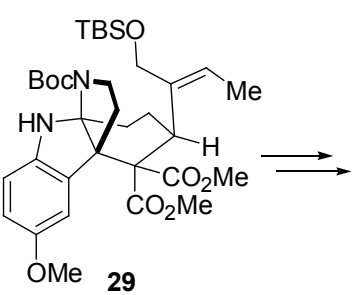

29

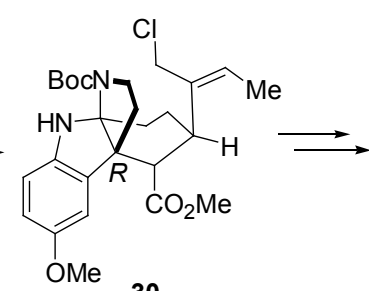

30

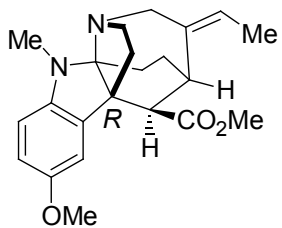

(-)-Vincorine

Scheme 4

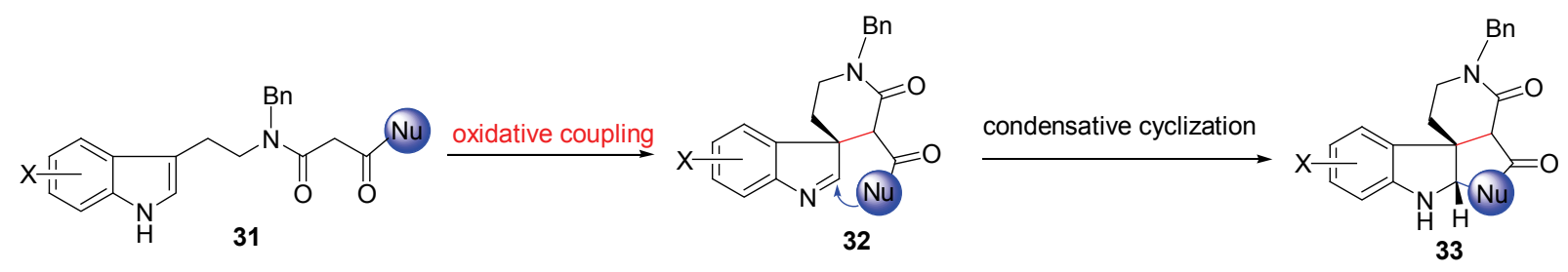

Scheme 5 
我们对反应适用底物范围进行了考察. 首先合成了有不 同取代基的吲哚酰胺，将之应用在氧化偶联/氧关环串 联反应中(表 1, Entries 1 7). 我们发现吲哚的电性对反 应的产率有一定的影响. 4 位或 5 位含有强给电子基团 的底物的反应产率一般比较低(表 1 , Entries 6,7 ), 而含 有吸电子基团的底物的反应产率都比较高(表 1, Entries 2 4, 9). 另外 4 位含有取代基的底物可能由于位阻作 用往往会阻碍氧化偶联反应的发生(回收部分底物), 从 而导致产率降低(表 1, Entries 3,7). 接下来我们对 $\beta$-酮 酸酸的取代基进行了改变. 我们发现 $\beta$ 位取代基对反应 的产率有较大的影响. 增大取代基位阻之后, 反应产率 大大降低(表 1, Entries 10１2). 反应中虽然底物完全消 失，但分离得到了第二步没有关环的产物(表 1, Entry $13)$, 说明位阻加大阻碍了第二步关环反应的发生.

表 $1 \beta$-酮酸酰胺 34 分子内氧化偶联/氧关环串联反应

Table 1 Intermolecular oxidative coupling/O-cyclization cascade reaction of $\beta$-ketoamide 34

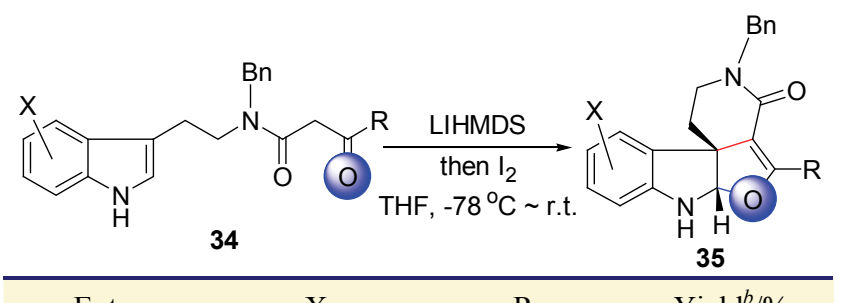

\begin{tabular}{cllc}
\hline Entry & \multicolumn{1}{c}{$\mathrm{X}$} & \multicolumn{1}{c}{$\mathrm{R}$} & Yield $^{b} \%$ \\
\hline 1 & $\mathrm{H}$ & $\mathrm{CH}_{3}$ & 74 \\
2 & $6-\mathrm{F}$ & $\mathrm{CH}_{3}$ & 87 \\
3 & $4-\mathrm{F}$ & $\mathrm{CH}_{3}$ & 66 \\
4 & $5-\mathrm{Br}$ & $\mathrm{CH}_{3}$ & 75 \\
5 & $7-\mathrm{MeO}$ & $\mathrm{CH}_{3}$ & 80 \\
6 & $5-\mathrm{MeO}$ & $\mathrm{CH}_{3}$ & $51^{c}$ \\
7 & $4-\mathrm{MeO}$ & $\mathrm{CH}_{3}$ & $38^{d}$ \\
8 & $6-\mathrm{Benzoxy}$ & $\mathrm{CH}_{3}$ & 76 \\
9 & $7-\mathrm{Cl}$ & $\mathrm{CH}_{3}$ & 78 \\
10 & $\mathrm{H}$ & $\mathrm{Benzyl}$ & 42 \\
11 & $\mathrm{H}$ & $n$-Butyl & 63 \\
12 & $\mathrm{H}$ & $t$-Butyl & $20^{e}$ \\
13 & $\mathrm{H}$ & Phenyl & 48 \\
\hline
\end{tabular}

${ }^{a}$ Reaction conditions: $34(0.2 \mathrm{mmol})$, LiHMDS (0.44 mmol), THF ( $\left.3 \mathrm{~mL}\right),-78$ ${ }^{\circ} \mathrm{C}, 30 \mathrm{~min}$, then addition of iodine $(0.22 \mathrm{mmol}),-78{ }^{\circ} \mathrm{C}$, then r.t., $30 \mathrm{~min}$. ${ }^{b}$ Isolated yield. ${ }^{c} 15 \%$ starting material was recovered. ${ }^{d} 22 \%$ starting material was recovered. ${ }^{e}$ Simple oxidative coupling product was isolated in $37 \%$ yield.

\section{4 分子内氧化偶联启动的串联反应在构建吡咯 并吲哚啉骨架中的应用}

通过 $\beta$-酮酸酰胺 34 分子内氧化偶联/氧关环串联反 应可以一步构建复杂的环吲哚啉环体系. 但从产物的结 构上看, 吲哚啉环并呋喃的结构在天然产物中不是很常 见. 而通过文献的调研, 我们发现在天然产物中, 吲哚 啉并吡咯环结构是很普遍的一种骨架, 而且这一类生物 碱都有较好的生物活性 ${ }^{[18]}$. 因此, 我们设想能否在分子
内引入氮原子，捕获分子内氧化偶联反应形成的亚胺， 从而可以一步构建多环的吲哚并吡咯环体系.

考虑到合成的方便性，我们设计了丙二酸酰胺 36 . 由于氮原子的亲核性受其保护基的影响，我们首先对氮 原子的保护基进行了考察(表 2, Entries 1 5). 可以看出 当没有保护基时，仅以 $21 \%$ 的收率得到关环产物. 而引 入烷基或芳基保护基后反应产率虽然有所提高, 但仍不 够理想(表 2, Entries 2,3). 最后我们发现吸电子保护基 可以极大地提高反应的产率，甲氧羰基和对甲苯磺基都 可以得到较好的产率(表 2, Entries 4, 5).

表 2 丙二酸酰胺 36 分子内氧化偶联/氮关环串联反应 Table 2 Intermolecular oxidative coupling/ $N$-cyclization cascade reaction of malonamide $\mathbf{3 6}$

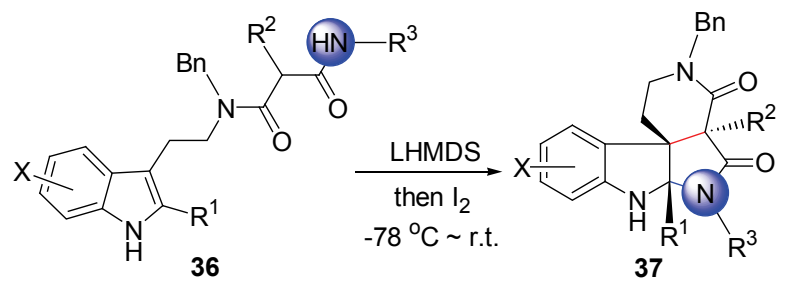

\begin{tabular}{cllllc}
\hline Entry & \multicolumn{1}{c}{$\mathrm{X}$} & $\mathrm{R}^{1}$ & $\mathrm{R}^{2}$ & \multicolumn{1}{c}{$\mathrm{R}^{3}$} & Yield/\% \\
\hline 1 & $\mathrm{H}$ & $\mathrm{H}$ & $\mathrm{H}$ & $\mathrm{H}$ & $21^{c}$ \\
2 & $\mathrm{H}$ & $\mathrm{H}$ & $\mathrm{H}$ & $\mathrm{Ph}$ & 43 \\
3 & $\mathrm{H}$ & $\mathrm{H}$ & $\mathrm{H}$ & $\mathrm{Bn}$ & 59 \\
4 & $\mathrm{H}$ & $\mathrm{H}$ & $\mathrm{H}$ & $\mathrm{CO}_{2} \mathrm{Me}$ & 80 \\
5 & $\mathrm{H}$ & $\mathrm{H}$ & $\mathrm{H}$ & $\mathrm{Ts}$ & 80 \\
6 & $6-\mathrm{F}$ & $\mathrm{H}$ & $\mathrm{H}$ & $\mathrm{CO}_{2} \mathrm{Me}$ & 61 \\
7 & $4-\mathrm{F}$ & $\mathrm{H}$ & $\mathrm{H}$ & $\mathrm{CO}_{2} \mathrm{Me}$ & 82 \\
8 & $5-\mathrm{Br}$ & $\mathrm{H}$ & $\mathrm{H}$ & $\mathrm{CO}_{2} \mathrm{Me}$ & 70 \\
9 & $4-\mathrm{MeO}$ & $\mathrm{H}$ & $\mathrm{H}$ & $\mathrm{CO}_{2} \mathrm{Me}$ & 87 \\
10 & $5-\mathrm{MeO}$ & $\mathrm{H}$ & $\mathrm{H}$ & $\mathrm{CO}_{2} \mathrm{Me}$ & 83 \\
11 & $7-\mathrm{MeO}$ & $\mathrm{H}$ & $\mathrm{H}$ & $\mathrm{CO}_{2} \mathrm{Me}$ & 80 \\
12 & $6-\mathrm{Benzoxy}$ & $\mathrm{H}$ & $\mathrm{H}$ & $\mathrm{CO}_{2} \mathrm{Me}$ & 87 \\
13 & $7-\mathrm{Cl}$ & $\mathrm{H}$ & $\mathrm{H}$ & $\mathrm{CO}_{2} \mathrm{Me}$ & 87 \\
14 & $5-\mathrm{Me}$ & $\mathrm{H}$ & $\mathrm{H}$ & $\mathrm{CO}_{2} \mathrm{Me}$ & 66 \\
15 & $\mathrm{H}$ & $\mathrm{Me}$ & $\mathrm{H}$ & $\mathrm{CO}_{2} \mathrm{Me}$ & 80 \\
16 & $\mathrm{H}$ & $\mathrm{Ph}$ & $\mathrm{H}$ & $\mathrm{CO}_{2} \mathrm{Me}$ & 77 \\
17 & $\mathrm{H}$ & $\mathrm{H}$ & $\mathrm{Me}$ & $\mathrm{CO}_{2} \mathrm{Me}$ & $0^{d}$ \\
18 & $\mathrm{H}$ & $\mathrm{H}$ & $\mathrm{Me}$ & $\mathrm{Ts}^{e}$ & $25^{e}$ \\
19 & $\mathrm{H}$ & $\mathrm{H}$ & $\mathrm{Ph}$ & $\mathrm{Ts}_{21}$ & 71 \\
20 & $\mathrm{H}$ & $\mathrm{Me}$ & $\mathrm{Ph}$ & $\mathrm{Ts}$ & 21 \\
\hline
\end{tabular}

${ }^{a}$ Reaction conditions: $36(0.2 \mathrm{mmol})$, LiHMDS $(0.44 \mathrm{mmol})$, THF $(3 \mathrm{~mL})$, $-78{ }^{\circ} \mathrm{C}, 30 \mathrm{~min}$, then addition of iodine $(0.22 \mathrm{mmol}),-78{ }^{\circ} \mathrm{C}$, then r.t., 30 min. ${ }^{b}$ Isolated yield. ${ }^{c} 30 \%$ dimerization product was isolated. ${ }^{d} 50 \%$ starting material was recovered. ${ }^{e} 7 \%$ starting material was recovered.

接下来我们对吲哚上的取代基进行了考察. 我们发 现和 $\mathrm{O}$ 关环反应不同, $\mathrm{N}$ 关环不受吲哚环的电性的影响 (表 2, Entries 6 14). 无论是吸电子还是给电子取代基, 都能以优秀的收率得到产物. 而且反应的收率也不受吲 哚 4 位的位阻影响(表 2, Entries 7,9). 而在吲哚环 2 位引 入取代基，同样可以较好的收率得到含有两个连续的季 
碳的多环骨架(表 2, Entries 15, 16). 我们进一步在考察 了丙二酸 $\alpha$ 位取代基对反应的影响. 在丙二酸的 $\alpha$ 位引 入一个甲基后, 完全不能分离得到产物, 只回收了部分 原料(表 2, Entry 17). 而把氮上保护基改为 Ts 后, 也仅 能以 $25 \%$ 收率得到关环产物(表 2, Entry 18). 而丙二酸 $\alpha$ 位为苯基时，反应产率大大提高(70\%，表 2, Entry 19). 由此可以看到, 氧化偶联反应不仅和丙二酸 $\alpha$ 的位阻有 关, 而且和其 $\mathrm{p} K_{\mathrm{a}}$ 有关. 最后我们同时在吲哚环 2 位和 丙二酸 $\alpha$ 位引入取代基, 发现同样可以得到关环产物. 虽然反应的产率偏低, 但一步构建了三个连续的季碳, 这在有机合成中是很少见的.

\section{5 总结与展望}

以含色胺的底物为原料, 我们发展了一类分子内氧 化偶联构建复杂吲哚生物碱骨架的方法. 首先通过分子 内氧化偶联反应, 我们成功构建了天然产物 Communesin 家族化合物的螺吲哚啉骨架, 从而完成了 (一)-Communesins A, B 和 F 的对映选择性合成. 而采用 分子内氧化偶联/关环的串联反应一步构建了复杂生物 碱 Vincorine 的多环骨架, 然后再经过五步完成了 (一)-Vincorine 的全合成. 通过对 Vincorine 的全合成, 我们展现了分子内氧化偶联启动的串联反应合成复杂 吲哚生物碱骨架的高效性. 为了快速, 多样地合成与天 然产物类似结构的小分子, 我们从易得的色胺衍生 $\beta$-酮 酸酰胺和丙二酸二酰胺为底物出发, 通过分子内氧化偶 联/关环串联反应, 一步构建了多环螺吲哚啉和多环吲 哚啉并吡咯骨架. 通过对复杂天然产物 Communesin 和 Vincorine 的合成, 证明了分子内氧化偶联反应是一种 非常有效的构建螺吲哚啉的季碳的方法. 同时通过其它 的亲核试剂捕获分子内氧化偶联反应生产的亚胺中间 体可以进一步启动后续的反应, 从简单的底物出发, 一 步构建含复杂环系骨架的分子, 这样可以大大提高全合 成的效率. 可以预见通过相同的策略在合成其它复杂生 物碱中应当能够得到广泛的应用.

\section{作者简介}

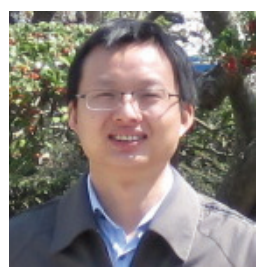

谢卫青副研究员于 2002 年毕业于兰州大学化学化 工学院, 2007 年在中国科学院上海有机化学研究所获得 博士学位. 2007 2009 年在马大为研究员小组任助理研 究员. 2009 2011 年在美国达拉斯西南医学中心进行博
士后研究. 2011 年, 在中国科学院上海有机化学研究所 马大为研究员小组任副研究员.

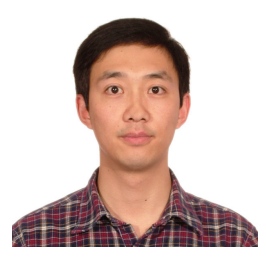

左智伟博士于 2007 年在南京大学获得学士学位, 之后进入中国科学院上海有机化学研究所攻读博士学 位. 于 2012 年在马大为研究员指导下获得博士学位. 博 士期间主要从事于复杂吲哚生物碱 Communesin 的全合 成研究.

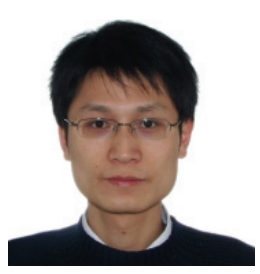

资伟伟博士于 2006 年毕业于兰州大学化学化工学 院，同年进入中国科学院上海有机所攻读博士学位，师 从马大为研究员. 博士期间致力于具有重要生物活性的 复杂天然产物 GB13 和 Vincorine 的全合成研究.

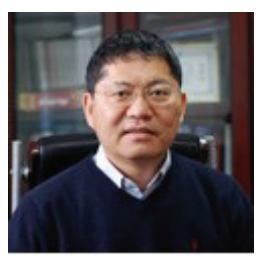

马大为研究员于 1984 年毕业于山东大学化学系, 1989 年于中国科学院上海有机化学研究所获博士学位. 1990～1994 年在美国匹兹堡大学化学系和 Mayo Clinic 神经化学和神经生物学研究部进行博士后研究. 研究方 向为化学生物学导向的有机合成及药物化学.

\section{References}

[1] Selected examples for oxidative homo-coupling: (a) Ivanoff, D.; Spassoff, A. Bull. Soc. Chim. Fr. 1935, $2,76$.

(b) Rathke, M. W.; Lindert, A. J. Am. Chem. Soc. 1971, 93, 4605.

(c) Brocksom, T. J.; Petragnani, N.; Rodrigues, R.; La Scala Teixeira, H. Synthesis 1975, 396.

(d) Frazier, R. H.; Harlow, R. L. J. Org. Chem. 1980, 45, 5408.

(e) Belletire, J. L.; Fry, D. F. J. Org. Chem. 1987, 45, 2549.

(f) Renaud, P.; Fox, M. A. J. Org. Chem. 1988, 45, 3745.

(g) Quermann, R.; Maletz, R.; Schafer, H. J. Liegigs Ann. Chem. 1993, 11, 1219.

(h) Kise, N.; Tokioka, K.; Aoyama, Y. J. Org. Chem. 1995, 60, 1100 .

(i) Langer, T.; Illich, M.; Felmchen, G. Tetrahedron Lett. 1995, 36, 
4409.

(j) Kim, J. W.; Lee, J. J.; Lee, S.-H.; Ahn, K.-H. Synth. Commun. 1998, 28, 1287.

[2] Selected examples for oxidative hetero-coupling: (a) Ito, Y.; Konoike, T.; Harada, T.; Saegusa, T. J. Am. Chem. Soc. 1977, 99, 1487.

(b) Baran, P. S.; Richter, J. M.; Lin, D. W. Angew. Chem., Int. Ed. 2005, 44, 609 .

(c) Baran, P. S.; DeMartino, M. P. Angew. Chem., Int. Ed. 2006, 45, 7083.

(d) Richter, J. M.; Whitefield, B.; Maimone, T. J.; Lin, D. W.; Castroviejo, P.; Baran, P. S. J. Am. Chem. Soc. 2007, 129, 12857.

(e) Richter, J. M.; Whitefield, B.; Maimone, T. J.; Lin, D. W.; Castroviejo, P.; Baran, P. S. J. Am. Chem. Soc. 2007, 129, 12857.

(f) DeMartino M. P.; Chen, K.; Baran, P. S. J. Am. Chem. Soc. 2008, 130, 11546.

[3] (a) Baran, P. S.; Richter, J. M. J. Am. Chem. Soc. 2004, 126, 7450.

(b) Baran, P. S.; Richter, J. M. J. Am. Chem. Soc. 2005, 127, 15394.

(c) Richter, J. M.; Whitefield, B.; Maimone, T. J.; Lin, D. W.; Castroviejo, P.; Baran, P. S. J. Am. Chem. Soc. 2007, 129, 12857.

(d) Baran, P. S.; Maimone, T. J.; Richter, J. M. Nature 2007, 446, 404.

(e) Richter, J. M.; Ishihara, Y.; Masuda, T.; Whitefield, B. W.; Llamas, T.; Pohjakallio, A.; Baran, P. S. J. Am. Chem. Soc. 2008, 130, 17938.

[4] (a) Baran, P. S.; Richter, J. M. J. Am. Chem. Soc. 2004, 126, 7450.

(b) Baran, P. S.; Richter, J. M. J. Am. Chem. Soc. 2005, 127, 15394.

(c) Baran, P. S.; Maimone, T. J.; Richter, J. M. Nature 2007, 446, 404.

(d) Richter, J. M.; Ishihara, Y.; Masuda, T.; Whitefield, B. W.; Llamas, T.; Pohjakallio, A.; Baran, P. S. J. Am. Chem. Soc. 2008, 130, 17938.

[5] (a) Martin, C. L.; Overman, L. E.; Rohde, J. A. J. Am. Chem. Soc. 2010, 132, 4894.

(b) Martin, C. L.; Overman, L. E.; Rohde, J. A. J. Am. Chem. Soc. 2008, 130, 7568 .

Fore review see: (c) Guo, F.; Clift, M. D.; Thomson, R. J. Eur. J. Org. Chem. 2012, 26, 4881.

For other examples see: (d) Li, Q.; Fan, A.; Lu, Z.; Cui, Y.; Lin, W.; Jia, Y. Org. Lett. 2010, 12, 4066.

(e) Li, Q.; Jiang, J.; Fan, A.; Cui, Y.; Jia, Y. Org. Lett. 2011, 13, 312.

[6] (a) Zuo, Z.; Xie, W.; Ma, D. J. Am. Chem. Soc. 2010, 132, 13226.

(b) Zuo, Z.; Ma, D. Angew. Chem., Int. Ed. 2011, 50, 12008.

[7] Zi, W.; Xie, W.; Ma, D. J. Am. Chem. Soc. 2012, $132,9126$.

[8] Fan, F.; Xie, W.; Ma, D. Org. Lett. 2012, 14, 1405.

[9] Fan, F.; Xie, W.; Ma, D. Chem. Commun. 2012, 48, 7571.

[10] (a) Numata, C.; Takahashi, Y.; Ito, T.; Takada, K.; Kawai, Y.; Usami, E.; Matsumura, M.; Imachi, T.; Ito, T.; Hasegawa, T. Tetrahedron Lett. 1993, 34, 2355.

(b) Jadulco, R.; Edrada, R. A.; Ebel, R.; Berg, A.; Schauman, K.; Wray, V.; Steube, K.; Proksch, P. J. Nat. Prod. 2004, 67, 78.

(c) Hayashi, H.; Matsumoto, H.; Akiyama, K. Biosci. Biotechnol. Biochem. 2004, 68, 753.

(d) Dalsgaard, P. W.; Blunt, J. W.; Munro, M. H. G.; Frisvad, J. C.; Christophersen, C. J. Nat. Prod. 2005, 68, 258.

[11] For synthetic studies see: (a) May, J. A.; Stoltz, B. M. Tetrahedron 2006, 62, 5262 .

(b) Crawley, S. L.; Funk, R. L. Org. Lett. 2006, 8, 3995.

(c) George, J. H.; Adlington, R. M. Synlett 2008, 14, 2093.
For total syntheses see Refs. [6] and (d) Yang, J.; Wu, H.; Shen, L.; Qin, Y. J. Am. Chem. Soc. 2007, 129, 13794.

(e) Liu, P.; Seo, J. H.; Weinreb, S. M. Angew. Chem. 2010, 122, 2044; Angew. Chem., Int. Ed. 2010, 49, 2000.

(f) Belmar, J.; Funk, R. L. J. Am. Chem. Soc. 2012, 134, 16941.

[12] (a) Smith, G. F. Chem. Ind. 1961, 1120.

(b) Wenkert, E.; Wickberg, B. J. Am. Chem. Soc. 1965, 87, 1580.

For an overview of the akuammiline alkaloid family, see: (c) Ramirez, A.; Garcia-Rubio, S. Curr. Med. Chem. 2003, 10, 1891.

[13] (a) Mokry, J.; Dubravkova, L.; Sefcovic, P. Experientia 1962, 18, 564.

(b) Das, B. C.; Cosson, J. P.; Lukacs, G.; Potier, P. Tetrahedron Lett. 1974, 15, 4229.

(c) Mamatas-Kalamaras, S.; Sevenet, T.; Thal, C.; Potier, P. Phytochemistry 1975, 14, 1637.

[14] Subramaniam, G.; Hiraku, O.; Hayashi, M.; Koyano, T.; Komiyama, K.; Kam, T. S. J. Nat. Prod. 2007, 70, 1783.

[15] (a) Schnoes, H.; Biemann, K.; Mokry, J.; Kompis, I.; Chatterjee, A.; Ganguli, G. J. Org. Chem. 1966, 31, 1641.

(b) Ahmad, Y.; Fatima, K.; Rahman, A.; Occolowitz, J.; Solheim, B.; Clardy, J.; Garnick, R.; Le Quesne, P. J. Am. Chem. Soc. 1977, 99, 1943.

[16] Cai, X. H.; Tan, Q. G.; Liu, Y. P.; Feng, T.; Du, Z. Z.; Li, W. Q.; Luo, X. D. Org. Lett. 2008, 10, 577.

[17] (a) Dounay, D. B.; Vollhardt, K. P. C. J. Am. Chem. Soc. 1990, 112, 5653.

(b) Lévy, J.; Sapi, J.; Laronze, J. Y.; Royer, D.; Toupet, L. Synlett 1992, 7, 601 .

(c) Dounay, A. B.; Overman, L. E.; Wrobleski, A. D. J. Am. Chem. Soc. 2005, 127, 10186.

(d) Shen, L.; Zhang, M.; Wu, Y.; Qin, Y. Angew. Chem., Int. Ed. 2008, 47, 3618 .

(e) Jones, S. B.; Simons, B.; Macmillan, D. W. C. J. Am. Chem. Soc. 2009, 131, 13606.

(f) Yasui, Y.; Kinugawab, T.; Takemoto, Y. Chem. Commun. 2009, 28,4275 .

(g) Zhang, M.; Huang, X.; Shen, L.; Qin, Y. J. Am. Chem. Soc. 2009, 131, 6013 .

(h) Zhang, D.; Song, H.; Qin, Y. Acc. Chem. Res. 2011, 44, 447.

(i) Zu, L.; Boal, B. W.; Garg, N. K. J. Am. Chem. Soc. 2011, 133, 8877 .

(j) Adams, G. L.; Caroll, P. J.; Smith, A. B. J. Am. Chem. Soc. 2012, 134, 4037.

[18] For reviews, see: (a) Anthoni, U.; Christophersen, C.; Nielsen, P. H. In Alkaloids: Chemical and Biological Perspectives, Vol. 13, Ed.: Pelletier, S. W., Pergamon, New York, 1999, p. 163.

(b) Cordell, G. A.; Saxton, J. E. In the Alkaloids: Chemistry and Physiology, Vol. 20, Eds.: Manske, R. H. F.; Rodrigo, R. G. A., Academic Press, New York, 1981, p. 3.

(c) Hino, T.; Nakagawa, M. In the Alkaloids: Chemistry and Pharmacology, Vol. 34, Ed.: Brossi, A., Academic Press, New York, 1989, p. 1.

(d) Sévenet, T.; Pusset, J. In the Alkaloids: Chemistry and Pharmacology, Vol. 48, Ed.: Cordell, G. A., Academic Press, New York, 1996, p. 1.

e) Steven, A.; Overman, L. E. Angew. Chem., Int. Ed. 2007, 46, 5488 .

(f) Ruiz-Sanchis, P.; Savina, S. A.; Albericio, F.; Álvarez, M. Chem. Eur. J. 2011, 17, 1388 . 